\title{
Amelioration of Collagen-induced Arthritis by CD95 (Apo-1/Fas)-ligand Gene Transfer
}

\author{
Haidi Zhang, ${ }^{*}$ Yiping Yang, ${ }^{*}$ Jennifer L. Horton, ${ }^{\star}$ Elena B. Samoilova, ${ }^{\star}$ Thomas A. Judge, ${ }^{\ddagger}$ Laurence A. Turka, ${ }^{\ddagger}$ \\ James M. Wilson, ${ }^{\star \ddagger}$ and Youhai Chen* \\ *Institute for Human Gene Therapy, Department of Molecular and Cellular Engineering; and ${ }^{*}$ Department of Medicine, University of \\ Pennsylvania School of Medicine, Philadelphia, Pennsylvania 19104
}

\begin{abstract}
Both rheumatoid arthritis and animal models of autoimmune arthritis are characterized by hyperactivation of synovial cells and hyperplasia of the synovial membrane. The activated synovial cells produce inflammatory cytokines and degradative enzymes that lead to destruction of cartilage and bones. Effective treatment of arthritis may require elimination of most or all activated synovial cells. The death factor Fas/Apo-1 and its ligand (FasL) play pivotal roles in maintaining self-tolerance and immune privilege. Fas is expressed constitutively in most tissues, and is dramatically upregulated at the site of inflammation. In both rheumatoid arthritis and animal models of autoimmune arthritis, high levels of Fas are expressed on activated synovial cells and infiltrating leukocytes in the inflamed joints. Unlike Fas, however, the levels of FasL expressed in the arthritic joints are extremely low, and most activated synovial cells survive despite high levels of Fas expression. To upregulate FasL expression in the arthritic joints, we have generated a recombinant replication-defective adenovirus carrying FasL gene; injection of the FasL virus into inflamed joints conferred high levels of FasL expression, induced apoptosis of synovial cells, and ameliorated collagen-induced arthritis in DBA/1 mice. The Fas-ligand virus also inhibited production of interferon- $\gamma$ by collagen-specific $T$ cells. Coadministration of Fas-immunoglobulin fusion protein with the Fasligand virus prevented these effects, demonstrating the specificity of the Fas-ligand virus. Thus, FasL gene transfer at the site of inflammation effectively ameliorates autoimmune disease. (J. Clin. Invest. 1997. 100:1951-1957.) Key words: gene therapy • adenovirus • apoptosis • autoimmune disease $\cdot$ CD95
\end{abstract}

Address correspondence to Youhai Chen, M.D., Ph.D., BRB-1 Rm. 401, Institute for Human Gene Therapy and Department of Molecular and Cellular Engineering, University of Pennsylvania School of Medicine, 422 Curie Blvd., Philadelphia, PA 19104. Phone: 215-8984671; FAX: 215-573-8606; E-mail: yhc@mail.med.upenn.edu

Received for publication 28 March 1997 and accepted in revised form 21 August 1997.

1. Abbreviations used in this paper: CIA, collagen-induced arthritis; CMV, cytomegalovirus.

J. Clin. Invest.

(C) The American Society for Clinical Investigation, Inc. 0021-9738/97/10/1951/07 \$2.00

Volume 100, Number 8, October 1997, 1951-1957

http://www.jci.org

\section{Introduction}

Collagen-induced arthritis (CIA) ${ }^{1}$ is an animal model for human rheumatoid arthritis. The disease can be induced in susceptible strains of animals by immunization with type II collagen $(1,2)$. Similar to rheumatoid arthritis, CIA is characterized by massive infiltration of synovial joints by inflammatory cells and hyperplasia of the synovial membrane. Both collagen-specific $\mathrm{T}$ and $\mathrm{B}$ lymphocytes are involved in the induction of CIA. The effector mechanisms that lead to joint tissue destruction are less well understood, and a number of inflammatory cell types have been implicated (2). These include fibroblast-like synoviocytes, bone marrow-derived macrophages, granulocytes, and dendritic cells as well as lymphocytes. Effective therapy of arthritis may require elimination or inhibition of all these cell types.

Fas:Fas-ligand interactions play pivotal roles in the death of cells of the immune system and the maintenance of immune tolerance to self antigens. Mutations of genes encoding Fas or FasL lead to lymphocytic proliferation and autoimmune inflammatory diseases (3-5). Fas is expressed constitutively in most tissues (5) and is dramatically upregulated at the site of inflammation $(6,7)$. In both rheumatoid arthritis and animal models of autoimmune arthritis, high levels of Fas are expressed on various inflammatory cells in the inflamed joints (7-10). Unlike Fas, however, expression of FasL is limited to a small number of cell types that include activated lymphocytes and immune privileged tissues. The former appears to use FasL to delete Fas-positive target cells, including self-reactive lymphocytes (11-14), whereas the latter may use FasL to maintain the immune privilege of such tissues as eye and testis $(15,16)$. Therefore, we hypothesized that FasL gene transfer at the site of inflammation might induce death of inflammatory cells and prevent unwanted tissue injury. We report here that adenovirus (Ad)-mediated FasL gene transfer in arthritic joints dramatically reduced inflammation and ameliorated collagen-induced arthritis in mice.

\section{Methods}

Generation of a recombinant adenovirus-carrying FasL gene. The AdFasL was generated based on human type 5 adenovirus with E1a, $\mathrm{E} 1 \mathrm{~b}$, and a portion of $\mathrm{E} 3$ region deleted. In brief, the plasmid (pAdlink) that contains sequences of $5^{\prime}$ inverted terminal repeat regions of Ad5, the cytomegalovirus enhancer and promotor, the simian virus 40 late gene polyadenylation signal, and sequences of Ad5 corresponding to 9.2-16.1 map units, were first purified. Mouse FasL cDNA (kindly provided by Dr. S. Nagata), which contains 32 bp of $5^{\prime}$ untranslated sequence, the entire coding sequence, and 55 bp of $3^{\prime}$ untranslated sequence, was inserted into pAd-link to generate pAdCMV-FasL. The recombinant virus was then produced by cotransfection of 293 cells with pAd-CMV-FasL- and ClaI/XbaI-digested Ad5 genomic DNA. Plaques of recombinant viruses were expanded and screened by PCR using FasL-specific primers. Recombinant virus 
carrying FasL gene was then amplified and purified through a cesium chloride gradient. In parallel experiments, a control Ad virus (designated as Ad vector) was also generated using previrus plasmid deleted of E1a and E1b that contains no FasL cDNA insert.

Reverse transcription (RT)-PCR. Total RNA was isolated using the Ultraspectrum RNA System ${ }^{\mathrm{TM}}$ (Biotecx Laboratories, Houston, TX) and RT-PCR was performed using the Promega Access System (Promega Corp., Madison, WI). The cDNA was synthesized for 45 min at $48^{\circ} \mathrm{C}$, and was amplified through $30-50$ cycles of reactions $\left(94^{\circ} \mathrm{C}\right.$ for $30 \mathrm{~s}, 55^{\circ} \mathrm{C}$ for $30 \mathrm{~s}$, and $72^{\circ} \mathrm{C}$ for $\left.1.5 \mathrm{~min}\right)$. The PCR products were then fractionated by electrophoresis on a $1 \%$ agarose gel, transferred to a nitrocellulose membrane, and blotted with full-length mouse FasL cDNA using the Genius ${ }^{\mathrm{TM}}$ Scaled-up Random Primed DNA Labeling Reaction System (Boehringer Mannheim Biochemicals, Indianapolis, IN). Color was developed using NBT and X-phosphate. The primers a and $\mathrm{b}$ correspond to the coding region sequence of FasL cDNA, which generates a 743-bp product (a: forward, 5'-ATGCAGCAGCCCATGAATT-ACCCA-3'; b: reverse, 5'-ATTGAATACTGCCCCCAGGTAGCT-3') (see Fig. $1 A$ ). The primers c and d correspond to the coding region sequence of FasL cDNA (forward primer) and poly A region sequence on viral vector (reverse primer), respectively, and generate a 668 -bp product representing transgenic FasL mRNA (c: forward, 5'-TCAGCTCTTCCACCTGCAG-3'; d: reverse, 5' -CACTGCATTCTAGTTGTGG-3') (see Fig. $1 A$ ).

Immunohistochemistry for Fas and FasL. Polyclonal rabbit antimouse Fas antibody was purchased from Santa Cruz Biotech. (Santa Cruz, CA). To investigate whether FasL protein can be detected by immunohistochemistry, we tested four anti-FasL antibodies (including one made in our laboratory) and two Fas-Ig fusion proteins. We found one rabbit polyclonal antibody (Santa Cruz Biotech.) that specifically stained Ad-FasL-infected Hela cells, but not Hela cells infected with control Ad vector (Zhang et al., unpublished data). This antibody was therefore used for this study. In brief, cryosections $(6-\mu \mathrm{m})$ of synovial tissues were treated with $1 \%$ hydrogen peroxide, blocked with $10 \%$ goat serum, and incubated with rabbit anti-Fas or anti-FasL antibody $(0.5 \mu \mathrm{g} / \mathrm{ml})$ for $1 \mathrm{~h}$ at room temperature. Biotinylated goat anti-rabbit IgG and avidin-peroxidase (Vector Laboratories, Inc., Burlingame, CA) were then added to the tissue. Counterstain was performed in $1 \%$ methyl green. Purified rabbit $\mathrm{Ig}(0.5 \mu \mathrm{g} / \mathrm{ml})$ was routinely used as controls.

Terminal deoxynucleotidyl transferase-mediated dUTP nick end labeling (TUNEL). For detection of apoptotic cells, ApoTag system was used (Oncor Inc., Gaithersburg, MD). In brief, synovial tissues were snap-frozen and cryosectioned $(6 \mu \mathrm{m})$. The 3 '-OH ends of fragmented DNA were labeled with digoxigenin-nucleotide using terminal deoxynucleotidyl transferase. The randomly incorporated nucleotide polymers were then detected by peroxidase-labeled antidigoxigenin antibody and the chromogen diaminobenzidin. Note that this technique detects strand breaks of DNA, but does not differentiate the types of the stimuli that lead to cell death.

Induction of the CIA. 6-8-wk-old male DBA/1 mice were purchased from Jackson Laboratory (Bar Harbor, ME) and housed in the University of Pennsylvania Animal Care Facilities. For CIA induction, mice were immunized by multiple intradermal injections of $100 \mu \mathrm{g}$ chicken type II collagen (Sigma Chemical Co., St. Louis, MO) in $100 \mu \mathrm{l}$ of $0.1 \mathrm{M}$ acetic acid emulsified in an equal volume of complete Freund's adjuvant containing $1 \mathrm{mg} / \mathrm{ml}$ of mycobacterium tuberculosis H37 RA (Difco Laboratories, Inc., St. Louis, MO). Mice were rechallenged with the same antigen preparation subcutaneously in the flank $21 \mathrm{~d}$ later. Disease develops $\sim 7-10 \mathrm{~d}$ after the second immunization.

Examination of the CIA. Mice were examined physically every other day in a blind fashion, and their paws were scored individually as follows (each paw received a separate score) (17): 0 , normal; 1 , Erythema and mild swelling confined to the ankle joint or toes; 2, Erythema and mild swelling extending from the ankle to the midfoot; 3 , Erythema and severe swelling extending from the ankle to the metatarsal joints; and 4, Ankylosing deformation with joint swelling.
These parameters correlate well to the histological changes in the arthritic joints. Severe cartilage destruction, bone erosion, and ankylosis are observed only in feet with a disease score of 3 and 4. Disease scores are presented and calculated as follows: the average disease score per mouse $=$ total disease scores from all the animals in the group divided by the number of animals. The maximum disease score per foot is the maximum disease score observed for each individual foot during the entire period of the experiment. The maximum disease score per foot is 4 , and the maximum disease score per mouse is 16. Since disease score data follow normal distribution, all statistical analyses were performed by ANOVA. For histological examination of the joint, mice were killed at different time points, and their paws were collected and fixed in $10 \%$ formalin. The paws were then decalcified in hydrochloric acid, embedded in paraffin, sectioned, and stained with hematoxylin and eosin.

Treatment of the CIA. FasL virus treatment was performed $3 \mathrm{~d}$ after disease onset ( $\sim 13 \mathrm{~d}$ after the second immunization). $10^{10}$ particles of Ad-FasL, Ad vector in $10 \mu \mathrm{l}$ of PBS, or PBS alone was injected intraarticularly to ankle joints $(5 \mu \mathrm{l})$ and periarticularly to tarsal joints $(5 \mu \mathrm{l})$ in both hind feet. Preliminary studies using rAd5 carrying Escherichia coli lac $Z$ gene showed that intraarticular injection led to lac $Z$ gene expression primarily inside of the ankle joints, whereas periarticular injection led to lac $Z$ gene expression in both the synovium and the perisynovial areas (Zhang et al., unpublished data).

Preparation of Fas-Ig fusion protein. BALB/c SCID mice (Jackson Laboratory) were injected subcutaneously with $10^{7}$ NIH3T3 cells that had been stably transfected with a construct consisting of the extracellular domain of mouse Fas and the $\mathrm{C} \gamma 1$ region of human $\mathrm{Ig}$ (kindly provided by Dr. Philip Leder, Harvard Medical School, Boston, MA) (14). Soluble Fas-Ig fusion protein was purified from the serum of SCID mice through protein-G affinity column as described (14).

\section{Results}

Construction of a recombinant adenovirus carrying Fas L gene (Ad-FasL). A recombinant adenovirus carrying the murine FasL gene (Fig. $1 A$ ) was generated by inserting the cloned FasL full-length cDNA into the plasmid pAd-link under the control of cytomegalovirus (CMV) promoter, followed by homologous recombination with human adenoviral DNA. The recombinant virus is replication-defective due to the deletion of $E 1 A$ and $E 1 B$ genes, and is capable of infecting a wide range of cell types in vitro. Fig. $1, B-C$ (lanes 1 and 2 ) illustrates Fas $L$ gene expression in Hela cells after Ad-FasL infection. Hela cells did not express detectable levels of FasL mRNA as determined by RT-PCR (lane 1); infection with Ad-FasL conferred FasL expression (lane 2). Similarly, human 293 cells did not express FasL after infection with control vector virus (Fig. $1 \mathrm{~F}$ ). High levels of FasL protein were detected on the surface of 293 cells after infection with rAd5-FasL virus (Fig. $1 G$ ).

FasL gene transfer in the arthritic joints. To test whether FasL gene can be expressed in the arthritic joints, we immunized DBA/1 mice with chicken type II collagen, and injected Ad-FasL intraarticularly and periarticularly in the hind feet. Immunization of DBA/1 mice with type II collagen induced arthritis of foot joints in virtually all the mice. Injection of AdFasL into the arthritic joints induced FasL mRNA expression as determined by RT-PCR (Fig. 1, $B$ and $C$, lane 3 ). No detectable levels of FasL mRNA was observed in arthritic joints injected with control Ad vector (Fig. 1, $B$ and $C$, lane 4). To ensure that FasL mRNA expressed was indeed encoded by the transgene, we performed RT-PCR using a $3^{\prime}$ primer encoding a 19-nucleotide sequence of the viral poly-A region. As shown 

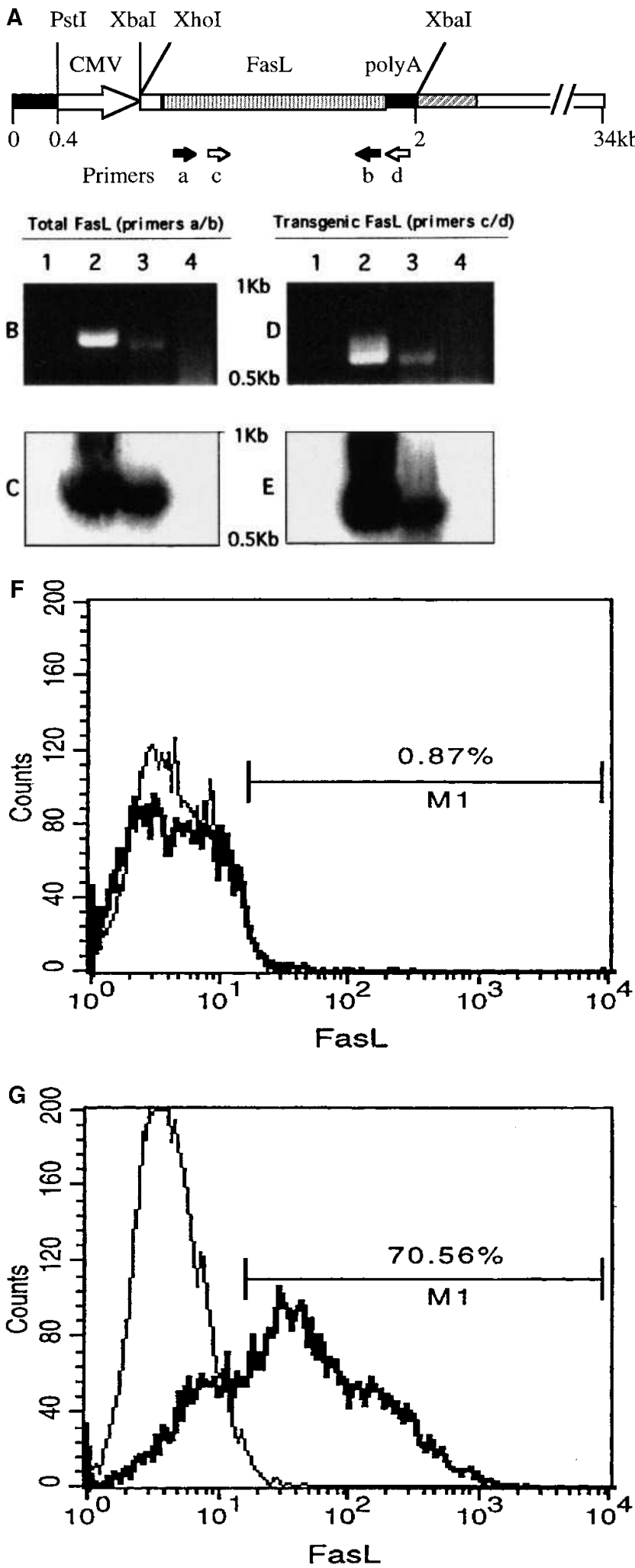

Figure 1. (A) The genomic organization of recombinant FasL-adenovirus. PstI, XbaI, and XhoI are representative restriction sites in the viral genome; $a-d$ are primers used for RT-PCR analyses of FasL mRNA. $(B-E)$ FasL mRNA expression as determined by RT-PCR. For preparation of Hela cells, $2 \times 10^{6}$ cells were infected with $10^{9}$ particles of Ad-FasL or control Ad viruses for $2 \mathrm{~d}$ in $20 \mathrm{ml}$ of DMEM containing $10 \%$ FBS. For preparation of arthritic joints, DBA/1 mice were immunized for arthritis with type II chicken collagen. Ad-FasL in Fig. 1, $D$ and $E$, both Ad-FasL transfected Hela cells (lane 2) and Ad-FasL-injected joint tissue (lane 3) expressed FasL mRNA encoded by the transgene. To test whether the FasL mRNA expressed was translated into protein in arthritic joints, we performed a series of immunohistochemical analyses using FasL-specific antibodies. As shown in Fig. $2 A$, little or no FasL was detected in arthritic joints treated with control Ad vector. By contrast, high levels of FasL protein were detected in the joints injected with Ad-FasL (Fig. 2 B).

Consistent with these observations, only a few apoptotic cells were detected in the arthritic joints treated with control Ad vector (Fig. $2 C$ ), whereas large numbers of apoptotic cells were seen in the joints injected with Ad-FasL (Fig. 2D). Apoptosis was observed in both intimal and subintimal regions of the inflamed synovium, and peaked $2-4 \mathrm{~d}$ after the FasL virus injection. By day 14, few apoptotic cells were detected, but dramatic reduction in synovial cell numbers was observed. The reduction in synovial cellularity occurred in the intimal and subintimal regions of the synovium as well as in the synovial cavity. Both activated synoviocytes and infiltrating leukocytes were affected. To determine whether the loss of synovial cells correlated to the levels of Fas expression, we performed immunohistochemistry of the synovia using anti-Fas antibodies. As shown in Fig. $2 E$, in the arthritic synovium, the intimal lining was made up of 5-8 layers of cells (a sign of hyperplasia) that expressed high levels of Fas; the subintimal region of the inflamed synovium was infiltrated by mononuclear cells that also expressed high levels of Fas. By contrast, in the arthritic synovium injected with the FasL virus, the cellularity and the levels of Fas expression were both dramatically reduced (Fig. 2 $F$ ). These results strongly suggest that high levels of functional FasL can be expressed in the arthritic joints using adenoviral vector, and that activated synovial cells are susceptible to FasL-induced apoptosis in vivo.

The effect of FasL gene transfer on collagen-induced arthritis. To test the effect of FasL expression on collagen-induced arthritis, we compared the disease course of mice treated with

or control Ad vector, $10^{10}$ particles each, were injected intraarticularly into the ankle joints $3 \mathrm{~d}$ after disease onset. Joint tissues were collected and snap-frozen in liquid nitrogen and analyzed for endogenous or transgenic FasL gene expression as described in Methods. All RT-PCR reactions were repeated for 50 cycles. RT-PCR of the joint tissues with 35 cycles also gave rise to positive, albeit weaker, signals (data not shown). ( $B$ and $D$ ) Ethidium bromide staining of fractionated DNA that had been amplified using primer pairs a/b and c/d, respectively. ( $C$ and $E$ ) Southern blot analysis of fractionated DNA that had been amplified using primer pairs $\mathrm{a} / \mathrm{b}$ and $\mathrm{c} / \mathrm{d}$, respectively. Lane 1, Hela cells infected with control Ad vector; lane 2, Hela cells infected with Ad-FasL; lane 3, arthritic ankle joints $3 \mathrm{~d}$ after injection of Ad-FasL; lane 4, arthritic ankle joints $3 \mathrm{~d}$ after injection of control Ad vector. ( $F$ and $G$ ) FasL protein expression as determined by flow cytometry. $10^{6} 293$ cells were infected with $10^{8}$ particles of Ad-FasL or control Ad viruses for $2 \mathrm{~d}$ in DMEM containing 10\% FBS. Cells were then harvested and stained with PE-labeled mouse anti-mouse FasL mAb (heavy line) (PharMingen, San Diego, CA) or control Ig (light line) per manufacturer's instructions. Fluorescence was acquired and analyzed on a Becton Dickinson FACScan using CellQuest software (Becton-Dickinson, San Jose, CA). Data presented represent 10,000 events. $(F)$ Cells were infected with control Ad virus. $(G)$ Cells were infected with rAd5-FasL. 

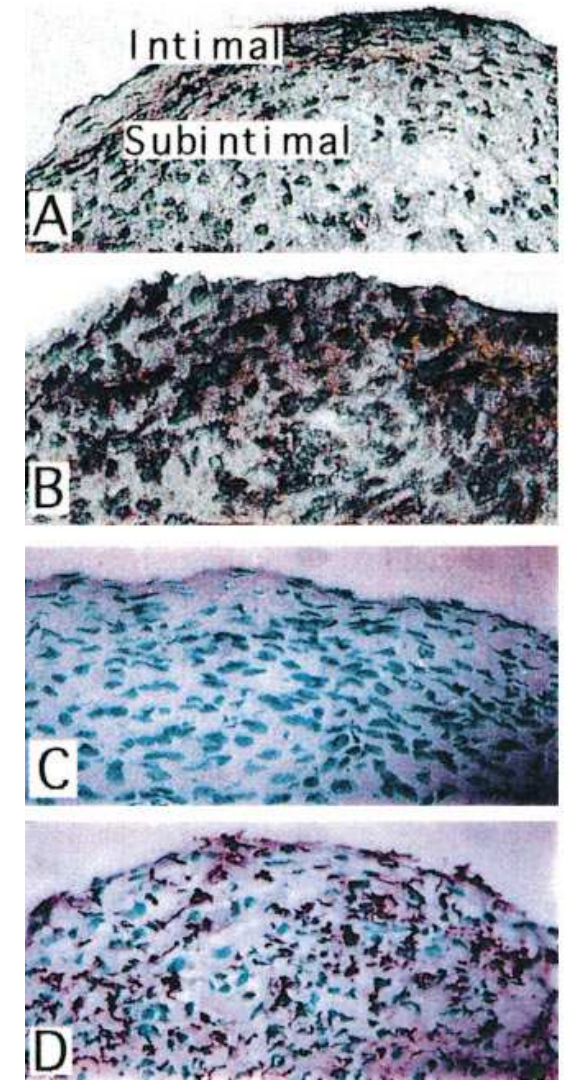
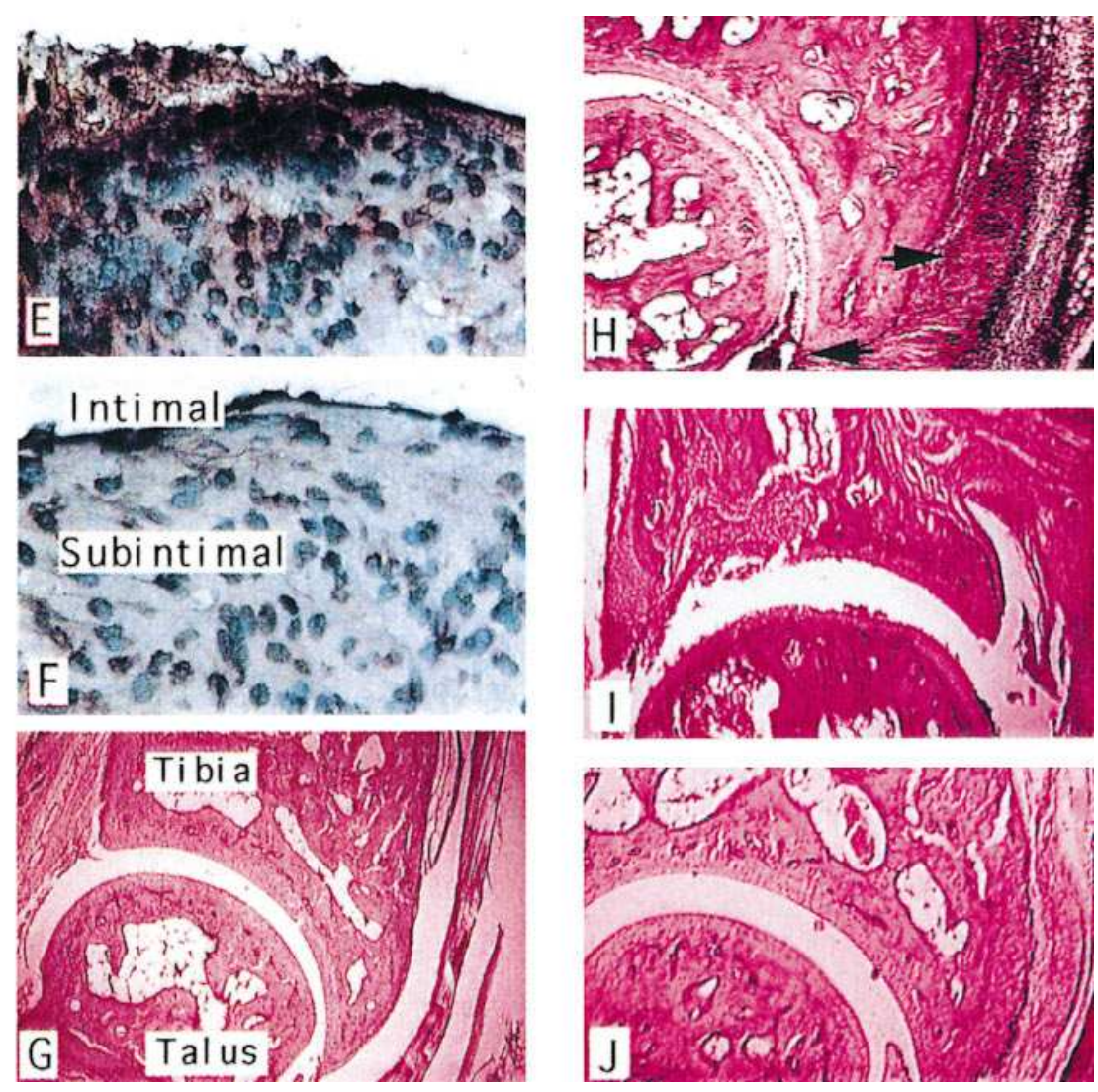

Figure 2. $(A$ and $B)$ FasL protein expression in arthritic joints. Two groups of DBA/1 mice, five mice per group, were immunized for arthritis with type II collagen. $3 \mathrm{~d}$ after disease onset, $10^{10}$ particles of Ad-FasL, or control Ad vector in $10 \mu \mathrm{l}$ of PBS, were injected intraarticularly and periarticularly to the ankle and tarsal joints of both hind feet. Synovial tissues of ankle joints were surgically removed and analyzed for FasL expression by immunohistochemistry. (A) Synovial tissue of ankle joint $3 \mathrm{~d}$ after injection of Ad vector (original magnification $150 \times$ ); $(B)$ Synovial tissue of ankle joint $3 \mathrm{~d}$ after injection of Ad-FasL (original magnification $150 \times)$. ( $C$ and $D$ ) Apoptosis in arthritic joints after FasL gene transfer. Synovial tissues were collected as in Fig. 2, $A$ and $B$ above and stained for apoptosis using Oncor ApopTag system (Oncor, Gaithersburg, MD). $(C)$ Synovial tissues of ankle joint $3 \mathrm{~d}$ after injection of Ad vector (original magnification $200 \times)$; $(D)$ synovial tissues of ankle joint $3 \mathrm{~d}$ after injection of Ad-FasL (original magnification $200 \times)$. $(E$ and $F$ ) Fas protein expression in the arthritic joints. Synovial tissues were collected as in Fig. 2, $A$ and $B$ above and analyzed for Fas expression by immunohistochemistry as described in Methods. $(E)$ Synovial tissues of ankle joint $6 \mathrm{~d}$ after disease onset (original, $400 \times$ ); $(F)$ synovial tissues of ankle joint $6 \mathrm{~d}$ after disease onset and $3 \mathrm{~d}$ after injection of FasL virus (original, $400 \times) .(G-J)$ Histological examination of arthritic joints after FasL gene transfer. DBA/1 mice, five mice per group, were immunized for arthritis with type II collagen. Three groups of DBA/1 mice, five mice per group, were immunized for arthritis with type II collagen. $3 \mathrm{~d}$ after disease onset, $10^{10}$ particles of Ad-FasL, or Ad vector in $10 \mu \mathrm{l}$ PBS, or PBS alone, was injected intraarticularly and periarticularly to ankle and tarsal joints in both hind feet. Mice were killed at different time points, and their paws were collected and fixed in $10 \%$ formalin. The paws were then decalcified in hydrochloric acid, embedded in paraffin, sectioned, and stained with hematoxylin and eosin. (G) Normal ankle joint before onset of the disease (original magnification $20 \times$ ). (H) PBS-injected ankle joint with a disease score of 3 (original magnification $40 \times$ ). Arrows indicate synovial infiltration and pannus formation. (I) Ad vector-injected ankle joint with a disease score of 4 (original magnification $20 \times$ ). $(J)$ Ad-FasLinjected ankle joint with a disease score of 1 (original magnification $40 \times$ ).

Ad-FasL with those treated with either PBS or control Ad vector. Both histopathology and clinical manifestations were used to monitor the disease. Fig. 2, $G-J$ are representative histological sections of the ankle joints. In both PBS and control Ad vector-treated groups, the arthritis is characterized by early massive leukocyte infiltration, synovitis, pannus formation (Fig. $2 H$ ), cartilage destruction, and bone erosion followed by remodeling and fibrosis (Fig. 2 I); by contrast, in the Ad-FasLtreated group, synovitis and synovial infiltration were dramatically diminished, and cartilage and bone erosion prevented (Fig. $2 J)$.

Fig. $3 A$ summarizes the disease course of 15 animals (presented as average disease score per mouse), and Fig. $3 B$ pre- sents the maximum disease score of individual feet. Arthritis developed in virtually all the mice and progressed rapidly in the PBS or control Ad vector-treated group. Intraarticular and periarticular injections of Ad-FasL $3 \mathrm{~d}$ after the onset of the disease effectively prevented the disease progression (Fig. $3 A$ ) and reduced significantly the severity of the inflammation (Fig. 3 B). Specifically, the maximum average disease score per mouse in PBS and Ad vector-treated groups were 4.6 \pm 3.1 and $4.2 \pm 3.4$, respectively (Fig. $3 A$, day 22 ). These were reduced to $2 \pm 2.2$ in the FasL virus-treated group (the variability in the data represents standard deviation; $P<0.01$ as determined by ANOVA). Similarly, the maximum disease score per foot in PBS and Ad vector-injected mice were 2.4 1.56 and 2.8 \pm 1.55 , 

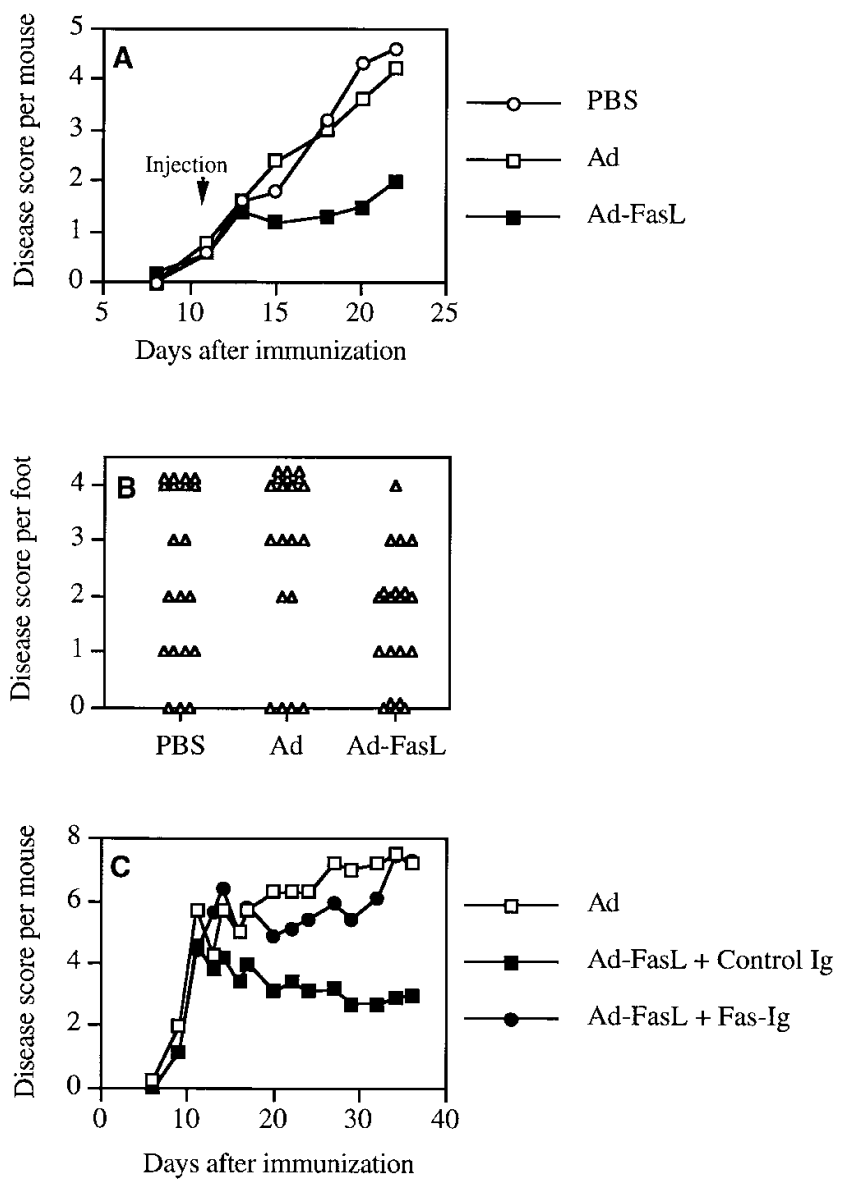

Figure 3. ( $A$ and $B$ ) Effect of FasL gene transfer on collagen-induced arthritis. Three groups of DBA/1 mice, five mice per group, were immunized for arthritis with type II collagen. $3 \mathrm{~d}$ after disease onset, $10^{10}$ particles of Ad-FasL, or Ad vector in $10 \mu \mathrm{l}$ of PBS, or PBS alone, was injected intraarticularly and periarticularly to ankle and tarsal joints in both hind feet. Mice were examined every other day for signs of joint inflammation and scored as described. $(A)$ Disease course presented as average disease score per mouse. $(B)$ Maximum disease scores of individual foot. A total of 20 feet per group are presented. The experiments were repeated three times with similar results. $(C)$ Effect of FasL-blockade on FasL gene transfer in collagen-induced arthritis. Three groups of DBA/1 mice, three to five mice per group, were immunized for arthritis with type II collagen. $3 \mathrm{~d}$ after disease onset, $10^{10}$ particles of Ad-FasL or Ad vector were injected intraarticularly and periarticularly to ankle and tarsal joints of both hind feet. $2 \mathrm{~d}$ later, mice that had received Ad-FasL were also injected intraarticularly and periarticularly with $15 \mu \mathrm{g}$ purified Fas-Ig or control human Ig (Jackson Biotech, West Chester, PA) in both hind feet. The Fas-Ig and control Ig injections were repeated every other day four times. Mice were examined for signs of joint inflammation, and were scored as described. $A d$, mice with injection of control Ad vector (three mice); Ad-FasL + control Ig, mice with injection of Ad-FasL and control Ig (five mice); Ad-FasL + Fas-Ig, mice with injection of Ad-FasL and Fas-Ig (five mice).

respectively (Fig. $3 B, 33 \mathrm{~d}$ after the second immunization); these were reduced to $1.5 \pm 1.17$ in the Ad-FasL-treated group $(P<0.01) .8$ out of 20 feet in the PBS-treated group and 10 out of 20 feet in the Ad vector-treated group developed severe joint swelling (disease score $=4$ ) followed by ankylosing deformation, whereas only 1 out of 20 feet in the Ad-FasL- treated group developed severe joint swelling (Fig. $3 B)(P<$ 0.0001). To examine whether the protective effect of Ad5FasL was due to FasL expression, we blocked FasL function by intraarticular and periarticular administration of Fas-immunoglobulin (Ig) fusion protein. As shown in Fig. $3 C$, intraarticular and periarticular injections of Fas-Ig four times significantly hindered the protective effect of Ad-FasL on CIA. Specifically, the average maximum disease score per mouse in control Ad vector treated group was 7.1 \pm 4.4 , and this was reduced to $3.0 \pm 1.5$ after injection of Ad-FasL $(P<0.05)$ (Fig. 3 $C$, day 38). Injection of Ad-FasL together with Fas-Ig prevented the effect of Ad-FasL (the maximum disease score was increased to $7.3 \pm 2.9$ after Fas-Ig injection, $P<0.007$ when compared with the Ad-FasL injected group).

The effect of FasL gene transfer on collagen-specific immune responses. The above results strongly suggest that transgenic expression of FasL in the arthritic joints induced apoptosis of inflammatory cells and diminished the progression of an ongoing autoimmune disease. One important question related to autoimmune disease therapy is whether transgenic expression of FasL at the site of inflammation also induces systemic tolerance of autoreactive cells. Therefore, we tested the immune responses to collagen in this arthritis gene therapy model. We confirmed reports that collagen-specific TH1 cells were activated in arthritic animals that were capable of secreting interferon (IFN)- $\gamma$ upon ex vivo stimulation with collagen $(2,18)$. Thus, large amounts of IFN- $\gamma$ were produced by inguinal lymph node cells of mice injected with either PBS or Ad vector (Fig. $4 H$ ). These were significantly reduced in mice injected with Ad-FasL. The reduction of IFN- $\gamma$ was restricted to collagen-specific T cells as concanavalin A-induced IFN- $\gamma$ secretion was not affected (Fig. 4). Furthermore, the reduction in IFN- $\gamma$ in Ad-FasL-treated mice was directly related to the FasL expression, since blockade of FasL with Fas-Ig fusion protein restored the IFN- $\gamma$ production (Zhang et al., unpublished data). In parallel experiments, we also tested anticollagen antibody responses in arthritic animals by collagen-specific ELISA. No significant differences were noted among mice treated with PBS, control Ad vector, or Ad-FasL within $40 \mathrm{~d}$ of viral injection (Zhang et al., unpublished data). This result may be due to the fact that viruses were injected $35 \mathrm{~d}$ after the first collagen immunization, and antibodies to collagen had already been generated well before Fas $L$ gene was expressed in the joints (Fig. 3).

\section{Discussion}

Both rheumatoid arthritis and animal models of autoimmune arthritis are characterized by hyperactivation of synovial cells and hyperplasia of synovial membrane. Activated synovial cells produce inflammatory cytokines and degradative enzymes that lead to destruction of cartilage and bones. Effective treatment of arthritis may require elimination of most or all activated synovial cells as well as arthritogenic lymphocytes that initiate joint inflammation. Although Fas is expressed at high levels on most synovial cells, FasL is expressed at extremely low levels (presumably by activated antigen-specific T cells), and most cells survive despite high levels of Fas expression (7, 10,19). To test whether upregulating FasL signal aids in diminishing the arthritic inflammation, we have generated a recombinant replication-defective adenovirus carrying Fas $L$ gene; injection of the FasL virus into inflamed joints conferred high 


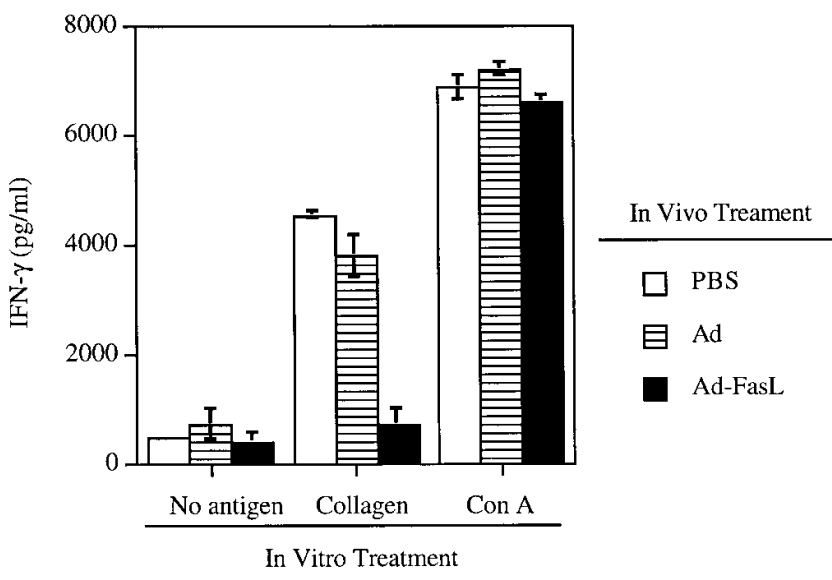

Figure 4. Expression of FasL in the arthritic joints inhibits IFN- $\gamma$ production by collagen-specific T cells. Mice were treated as in Fig. 3, $A$ and $B$, and were killed $40 \mathrm{~d}$ after the second immunization. Inguinal lymph nodes were harvested, and single-cell suspensions were prepared. Lymph node cells, $10^{6}$ each, were cultured in $0.2 \mathrm{ml}$ of serumfree medium (X-vivo 20; Biowhittacker, Walkersville, MD) with or without $10 \mu \mathrm{g} / \mathrm{ml}$ of collagen or $2.5 \mu \mathrm{g} / \mathrm{ml}$ of Con-A. Culture supernatants were collected $40 \mathrm{~h}$ later, and IFN- $\gamma$ concentrations were determined by sandwich ELISA as we described (41). The anti-IFN- $\gamma$ mAb R4-6A2 was used as coating antibody, and biotinylated antiIFN- $\gamma$ mAb XMG1.2 (PharMingen, San Diego, CA) was used as detecting antibody (41). Each data point represents a mean \pm SD from five mice. The experiments were repeated twice with similar results. The differences in collagen-induced IFN- $\gamma$ production between the Ad-FasL-treated group and PBS or Ad vector-treated groups were statistically significant $(P<0.01$ as determined by ANOVA). Open bar, mice treated with PBS; striped bar, mice treated with control Ad vector; filled bar, mice treated with Ad-FasL.

levels of FasL expression, induced apoptosis of synovial cells, and ameliorated the disease.

Compared to other forms of arthritis therapy, gene therapy offers a number of unique advantages (20-23). Cell membrane proteins such as FasL can be readily expressed; protein expression can be targeted to the site of the lesion and can last for a relatively long period of time. Initially, gene delivery to joint tissues was achieved by intraarticular injection of genetically modified cells $(20,24,25)$. In these studies, synoviocytes were treated ex vivo with recombinant retrovirus carrying the gene of interest, and were then injected back into the synovial cavity. This method was shown to be effective in delivering IL-1 antagonist gene and in inhibiting arthritic inflammation (20, 24-26). Recently, we and others have been investigating direct gene delivery into synovial joints using replication-defective adenoviral vector (27) or retroviral vector (22). Adenoviral vectors were shown to be extremely effective in direct intraarticular gene transfer, presumably due to their capacity to infect quiescent cells and to persist in stable episomic state in a variety of cell types $(28,29)$. Intraarticular administration of recombinant adenovirus led to high levels of transgene expression in the synovium, which lasted a minimum of 2-4 wk (27). Intraarticular injection of recombinant retrovirus into inflamed joints (where proliferating cells were present) also led to significant intraarticular transgene expression (22). As transgene expression was localized primarily inside of the treated joints, systemic toxicity of the transgene could be significantly minimized. In the course of our current studies, no systemic toxicity was ever observed in mice intraarticularly and periarticularly injected with Ad5-FasL, and histochemical analyses of the liver, spleen, and thymus revealed no increased apoptosis or tissue injury. By contrast, intravenous injection of $10^{10}$ particles of FasL virus induced significant apoptosis in the liver and the spleen as determined by TUNEL analyses (Zhang et al., unpublished data).

Although transgene expression lasts for weeks (as opposed to hours or days when the protein is injected), none of the current generations of adenoviral vectors can sustain long-term gene expression $(30,31)$. This finding is partially due to the fact that adenoviral vectors are potentially immunogenic, and that both viral-specific $\mathrm{T}$ and $\mathrm{B}$ cell responses are elicited in the recipients treated with recombinant adenoviruses (30, 32, 33). These problems may be partially resolved by using newer generations of adenoviral vectors that are further deleted of structural genes encoding viral antigens (34), and by coadministration of immune-suppressive agents or costimulation blocking reagents $(31,35)$. A major goal of our current investigation is to determine whether FasL gene expression at the site of gene transfer also induces apoptosis of vector-specific lymphocytes so that the immune barrier impeding gene therapy can be eliminated. In this study, we noted that transgene expression after intraarticular and periarticular administration of recombinant adenovirus was localized primarily inside of the inflamed joints, which lasted a minimum of $10 \mathrm{~d}$. As FasL gene transfer may also induce death of FasL-expressing cells by Fasmediated apoptosis, however, the length of FasL expression in the joints may not be used to measure the efficiency of adenoviral vector-mediated gene transfer. Experiments are underway to study adenoviral vector-mediated gene transfer in FasL virus-treated mice using a replication-defective adenovirus carrying E. coli LacZ gene.

The roles of Fas/FasL in autoimmunity and transplant rejection have been vigorously investigated $(5,11,36,37)$. Deficiency in Fas/FasL function leads to systemic autoimmunity (3-5), whereas provision of FasL signal prevents allograft rejection and confers immune privilege $(15,16,37)$. By contrast, the role of FasL in antitumor immunity is less clear. While expression of FasL on human tumor cells were found to evade immune attack and confer immune privilege $(38,39)$, a recent report using murine tumor cells suggests that FasL expression may also enhance antitumor immunity (40). This discrepancy may stem from the differences in the cell types used and in the levels of Fas/FasL expression, as well as the cytokine milieu of the microenvironment. In both rheumatoid arthritis and animal models of autoimmune arthritis, Fas expression is greatly upregulated (7-9). Intraarticular injection of anti-Fas $\mathrm{mAb}$ inhibits development of arthritis in HTLV-I tax transgenic mice (7). To investigate whether FasL is expressed in the arthritic joints, we performed RT-PCR (for FasL mRNA) as well as immunohistochemistry (for FasL protein) of the arthritic synovium; little or no FasL signal was detected in the arthritic synovium regardless of the methods used (Fig. 1, $B$ and $C$, and Fig. $2 A$ ). Similar results were reported by Cantwell et al. for rheumatoid arthritis in which neither FasL protein nor FasL mRNA was detected in the inflamed synovium (19). Thus, the level of endogenous FasL expressed in the arthritic joints may be extremely low, which may not be sufficient to eliminate inflammatory cells through apoptosis. Whether this deficiency in 
FasL-mediated apoptosis plays a causal role in the development of autoimmune arthritis is unclear.

In summary, our data suggest that transgenic expression of FasL in the arthritic joints by somatic gene transfer prevents progression of the disease, and diminishes tissue injury. Thus, FasL gene transfer may serve as a novel form of therapy for chronic inflammatory diseases such as rheumatoid arthritis.

\section{Acknowledgments}

We thank Drs. Shigekazu Nagata, Philip Leder, Shyr-Te Ju, and Howard L. Weiner for reagents, and Qin Su, Kazu Tsukamoto and Morphology Core for technical assistance.

This work was supported in part by grants from the Cystic Fibrosis Foundation and Genovo Inc., a company that Dr. Wilson founded, and in which he holds equities.

\section{References}

1. David, C.S. 1990. Genes for MHC, TCR and MIs determine susceptibility to collagen induced arthritis. APMIS. 98:575-584.

2. Holmdahl, R., M. Andersson, T.J. Goldschmidt, K. Gustafsson, L. Jansson, and J.A. Mo. 1990. Type II collagen autoimmunity in animals and provocations leading to arthritis. Immunol. Rev. 118:193-232.

3. Nagata, S., and T. Suda. 1995. Fas and Fas ligand: lpr and gld mutations. Immunol. Today. 16(1):39-43.

4. Cohen, P.L., and R.A. Eisenberg. 1992. The lpr and gld genes in systemic autoimmunity: life and death in the Fas lane. Immunol. Today. 13(11):427-428.

5. Nagata, S., and P. Golstein. 1995. The Fas death factor. Science (Wash. DC). 267:1449-1456.

6. Tanaka, H., K. Ota, M. Ikusaka, M. Ejima, and S. Maruyama. 1995. Expression of Fas-antigen on T cells in multiple sclerosis. Rinsho Shinkeigaku. 35:299301.

7. Fujisawa, K., H. Asahara, K. Okamoto, H. Aono, T. Hasunuma, T. Kobata, Y. Iwakura, S. Yonehara, T. Sumida, and K. Nishioka. 1996. Therapeutic effect of the anti-Fas antibody on arthritis in HTLV-1 tax transgenic mice. $J$. Clin. Invest. 98(2):271-278.

8. Nakajima, T., H. Aono, T. Hasunuma, K. Yamamoto, T. Shirai, K. Hirohata, and K. Nishioka. 1995. Apoptosis and functional Fas antigen in rheumatoid arthritis synoviocytes. Arthritis Rheum. 38(4):485-491.

9. Tsuboi, M., K. Eguchi, A. Kawakami, N. Matsuoka, Y. Kawabe, T. Aoyagi, K. Maeda, and S. Nagataki. 1996. Fas antigen expression on synovial cells was down-regulated by interleukin 1 beta. Biochem. Biophys. Res. Commun. 218(1):280-285.

10. Firestein, G.S., M. Yeo, and N.J. Zvaifler. 1995. Apoptosis in rheumatoid arthritis synovium. J. Clin. Invest. 96(3):1631-1638.

11. Singer, G.G., and A.K. Abbas. 1994. The Fas antigen is involved in peripheral but not thymic deletion of $\mathrm{T}$ lymphocytes in $\mathrm{T}$ cell receptor transgenic mice. Immunity. 1:365-371.

12. Brunner, T., R.J. Mogil, D. LaFace, N.J. Yoo, A. Mahboubi, F. Echeverri, S.J. Martin, W.R. Force, D.H. Lynch, C.F. Ware, and D.R. Green. 1995. Cell-autonomous Fas (CD95)/Fas-ligand interaction mediates activation-induced apoptosis in T-cell hybridomas. Nature (Lond.). 373:441-444.

13. Dhein, J., H. Walczak, C. Baumler, K.-M. Debatin, and P.H. Krammer. 1995. Autocrine T-cell suicide mediated by APO-1/(Fas/CD95). Nature (Lond.). 373:438-441.

14. Ju, S.T., D.J. Panka, H. Cul, R. Ettinger, M. El-Khatib, D.H. Sherr, B.Z. Stanger, and A. Marshak-Rothstein. 1995. Fas(CD95)/FasL interaction required for programmed cell death after T-cell activation. Nature (Lond.). 373: $444-448$.

15. Griffith, T.S., T. Brunner, S.M. Fletcher, D.R. Green, and T.A. Ferguson. 1995. Fas ligand-induced apoptosis as a mechanism of immune privilege. Science (Wash. DC). 270:1189-1192.

16. Bellgrau, D., D. Gold, H. Selawry, J. Moore, A. Franzusoff, and R.C. Duke. 1995. A role for CD95 ligand in preventing graft rejection. Nature (Lond.). 377(6550):630-632.

17. Myers, L.K. 1993. Collagen-induced arthritis. In Current Protocols in Immunology. J.E. Coligan et al., editors. Sarah Greene, New York. 15.5.1-24.

18. Myers, L.K., J.M. Seyer, J.M. Stuart, K. Terato, C.S. David, and A.H. Kang. 1993. T cell epitopes of type II collagen that regulate murine collagen- induced arthritis. J. Immunol. 151(1):500-505.

19. Cantwell, M.J., T. Hua, J. Zvaifler, and T.J. Kipps. 1996. CD95 and Fasligand expression and apoptosis in rheumatoid arthritis. Arthritis Rheum. 39(9): S74

20. Evans, C.H., and P.D. Robbins. 1995. Progress toward the treatment of arthritis by gene therapy. Ann. Med. 27(5):543-546.

21. Bandara, G., G.M. Mueller, J. Galea-Lauri, M.H. Tindal, H.I. Georgescu, M.K. Suchanek, G.L. Hung, J.C. Glorioso, P.D. Robbins, and C.H. Evans. 1993. Intraarticular expression of biologically active interleukin 1-receptor-antagonist protein by ex vivo gene transfer. Proc. Natl. Acad. Sci. USA. 90(22):10764-10768.

22. Makarov, S.S., J.C. Olsen, W.N. Johnston, J.H. Schwab, S.K. Anderle, R.R. Brown, and J.S. Haskill. 1995. Retrovirus mediated in vivo gene transfer to synovium in bacterial cell wall-induced arthritis in rats. Gene Ther. 2(6):424428

23. Doherty, P.J. 1995. Gene therapy and arthritis. J. Rheumatol. 22(7): $1220-1223$.

24. Hung, G.L., J. Galea-Lauri, G.M. Mueller, H.I. Georgescu, L.A. Larkin, M.K. Suchanek, M.H. Tindal, P.D. Robbins, and C.H. Evans. 1994. Suppression of intra-articular responses to interleukin-1 by transfer of the interleukin-1 receptor antagonist gene to synovium. Gene Ther. 1(1):64-69.

25. Makarov, S.S., J.C. Olsen, W.N. Johnston, S.K. Anderle, R.R. Brown, A.S. Baldwin, Jr., J.S. Haskill, and J.H. Schwab. 1996. Suppression of experimental arthritis by gene transfer of interleukin 1 receptor antagonist cDNA. Proc. Natl. Acad. Sci. USA. 93(1):402-406.

26. Otani, K., I. Nita, W. Macaulay, H.I. Georgescu, P.D. Robbins, and C.H. Evans. 1996. Suppression of antigen-induced arthritis in rabbits by ex vivo gene therapy. J. Immunol. 156(9):3558-3562.

27. Roessler, B.J., E.D. Allen, J.M. Wilson, J.W. Hartman, and B.L. Davidson. 1993. Adenoviral-mediated gene transfer to rabbit synovium in vivo. $J$. Clin. Invest. 92(2):1085-1092. 692

28. Wilson, J.M. 1993. Vehicles for gene therapy. Nature (Lond.). 365:691-

29. Casscells, W., and J.T. Willerson. 1995. Amphotropic but not atherotropic: another caveat for adenoviral gene therapy. J. Clin. Invest. 95(6):24252426

30. Yang, Y., Q. Li, H.C.J. Ertl, and J.M. Wilson. 1995. Cellular and humoral immune responses to viral antigens create barriers to lung-directed gene therapy with recombinant adenoviruses. J. Virol. 69(4):2004-2015.

31. Yang, Y., Q. Su, I.S. Grewal, R. Schilz, R.A. Flavell, and J.M. Wilson. 1996. Transient subversion of CD40 ligand function diminishes immune responses to adenovirus vectors in mouse liver and lung tissues. J. Virol. 70(9): 6370-6377.

32. Engelhardt, J.F., Y. Yang, L.D. Stratford-Perricaudet, E.D. Allen, K. Kozarsky, M. Perricaudet, J.R. Yankaskas, and J.M. Wilson. 1993. Direct gene transfer of human CFTR into human bronchial epithelia of xenografts with E1deleted adenoviruses. Nat. Genet. 4:27-34

33. Yang, Y., H.C.J. Ertl, and J.M. Wilson. 1994. MHC class I restricted cytotoxic T lymphocytes to viral antigens destroy hepatocytes in mice infected with E1 deleted recombinant adenoviruses. Immunity. 1:433-442.

34. Yang, Y., F.A. Nunes, K. Berencsi, E. Gonczol, J.F. Engelhardt, and J.M. Wilson. 1994. Inactivation of E2a in recombinant adenoviruses improves the prospect for gene therapy in cystic fibrosis. Nat. Genet. 7(3):362-369.

35. Dai, Y., E.M. Schwarz, D. Gu, W.W. Zhang, N. Sarvetnick, and I.M. Verma. 1995. Cellular and humoral immune responses to adenovirus vectors containing factor IX gene: tolerization of factor IX and vector antigens allows for long-term expression. Proc. Natl. Acad. Sci. USA. 92(5):1401-1405.

36. Russell, J.H., B. Rush, C. Weaver, and R. Wang. 1993. Mature T cells of autoimmune $\mathrm{lpr} / \mathrm{lpr}$ mice have a defect in antigen-stimulated suicide. Proc. Natl. Acad. Sci. USA. 90(10):4409-4413.

37. Lau, H.T., M. Yu, A. Fontana, and C.J. Stoeckert, Jr. 1996. Prevention of islet allograft rejection with engineered myoblasts expressing FasL in mice. Science (Wash. DC). 273(5271):109-112.

38. Hahne, M., D. Rimoldi, M. Schroter, P. Romero, M. Schreier, L.E. French, P. Schneider, T. Bornand, A. Fontana, D. Lienard, et al. 1996. Melanoma cell expression of Fas (Apo-1/CD95) ligand: implications for tumor immune escape. Science (Wash. DC). 274:1363-1366.

39. Strand, S., W.J. Hofmann, H. Hug, M. Muller, G. Otto, D. Strand, S.M. Mariani, W. Stremmel, P.H. Krammer, and P.R. Galle. 1996. Lymphocyte apoptosis induced by $\mathrm{CD} 95$ (Apo-1/Fas) ligand-expressing tumor cells-a mechanism of immune evasion? Nat.Med.2(12):1361-1366.

40. Seino, K., N. Kayagaki, K. Okumura, and H. Yagita. 1997. Antitumor effect of locally produced CD95 ligand. Nat. Med. 3(2):165-170.

41. Chen, Y., J.-I. Inobe, and H.L. Weiner. 1995. Induction of oral tolerance to MBP in CD8-depleted mice. J. Immunol. 155:910-916. 